\section{Non-adjacent Sacral Dural Arteriovenous Fistula and Thoracolumbar Spinal Arteri- ovenous Malformation}

\section{Sir,}

A 42-year female presented with a 3-month history of bilateral leg weakness and diminished sensation. Digital subtraction angiography (DSA) showed a sacral dural arteriovenous fistula (sDAVF), which was supplied via the right lateral sacral artery. The fistula, located at the level of L5-S1, was drained via the posterior spinal vein. Spinal magnetic resonance imaging (MRI) confirmed same finding. The patient underwent a right L5-S1 sDAVF embolisation on admission, and moderate post-procedure clinical improvement was noted. Interventional therapy improved her bilateral lower extremity numbness, and muscle strength improved the following day. Three months later, our patient returned to hospital complaining of bilateral lower extremity skin hypersensitivity. Her bilateral muscle strength was graded 4+. DSA findings showed no recanalisation of the original fistula post-admission. Spinal DSA subsequently confirmed an arteriovenous malformation (AVM) at L1 that was supplied via anterior spinal artery. After careful evaluation, we undertook conservative treatment, and her symptoms resolved.

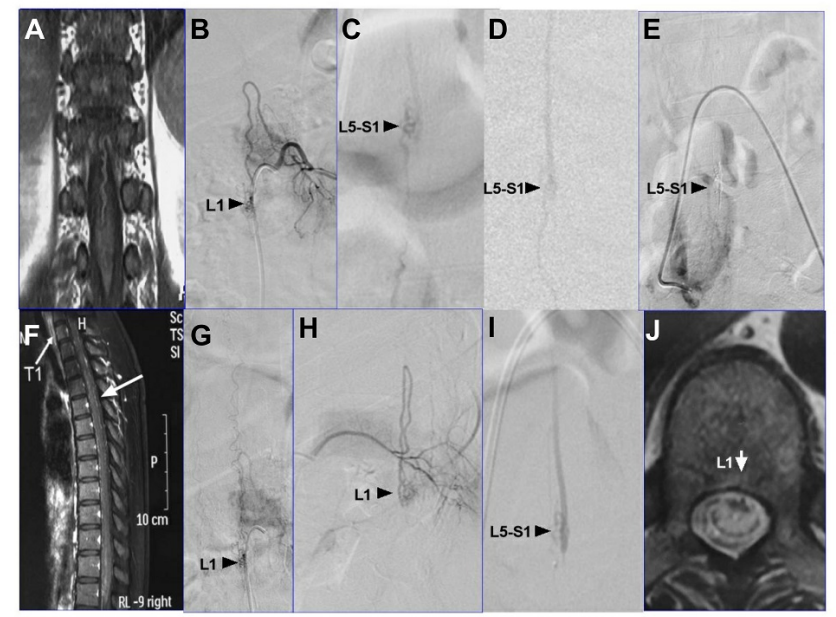

Figure 1: (A) Preoperative coronal T1-weighted MRI scan. (B) Angiography shows an AVM (arrow) located T12-L1 by an anterior spinal artery at second hospitalisation. (C) Angiography shows an arteriovenous fistula (arrow) located at L5-S1 at first admission. (D) Preoperative sagittal DSA scan of (C). (E) Angiography indicates no recurrence after embolisation. (F) MRI (sagittal view, T1 weighted image) confirmed that the drainage vein was not consistent with the DSA findings in the thoracolumbar spine (B). (G, H) Angiography showed that the shape of drainage vein was consistent with the result of MRI examination (A). (I) The DSA showed that the fistula was located at L5-S1 by first hospitalisation. (J) MRI (T1 axial images) showed AVMs located around at L1.
Spinal AVFs are classified as paraspinal, epidural, dural, or intradural fistulas. ${ }^{1,2}$ For intradural fistulas, sDAVFs alone are most common, and concomitant lesions have not been commonly reported. ${ }^{3,4}$ sDAVF malformations associated with spinal AVMs are relatively rare. We found that DSA findings were not consistent with the MRI findings (Figure $A$ ); therefore, a diagnosis in the T12-L1 AVM segment was missed (Figure $B \& C$ ) and we only applied an L5-S1 sDAVF embolization (Figure D \& E). Based on location and shape, when DSA and MRI findings were combined, we considered that the sDAVF was supplied through the right lateral sacral artery and that the AVM was supplied through the L1 anterior spinal artery (Figure B). It is important to determine whether the shape of the supplying artery is consistent between DSA and MRI examinations. The shape of the supplying artery for the L5-S1 SDAVF, observed using DSA, was not consistent with the shape observed on MRI findings in the first admission period (Figure F, G, H\&I). Moreover, the vein shape and the DSA location (L5-S1) differed from that observed on MRI (T8-12) (Figure I \& J). The location of the AVM and the AVF were not consistent using DSA and MRI examinations.

To avoid a missed diagnosis, multimodal imaging should be performed, and the consistency of the arterial blood supply, position of the malformed section, and shape of the drainage vein should be evaluated, to avoid the misdiagnosis of concomitantAVM.

\section{CONFLICT OF INTEREST:}

Authors declared no conflict of interest.

\section{AUTHORS' CONTRIBUTION:}

GZ: Drafted the manuscript.

FS: Edited and revised the manuscript.

\section{REFERENCES}

1. Koch MJ, Stapleton CJ, Agarwalla PK, Torok C, Shin JH, Coumans JV, et al. Open and endovascular treatment of spinal dural arteriovenous fistulas: A 10-year experience. J Neuro Surg Spine 2017; 26(4):519-23. doi: 10.3171/ 2016.9.SPINE16394.

2. Li J, Li G, Bian L, Hong T, Yu J, Zhang H, et al. Concomitant lumbosacral perimedullary arteriovenous fistula and spinal dural arteriovenous fistula. World neurosurgery. 2017; 105:1041. doi: 10.1016/j.wneu.2017.06.149.

3. Rosi A, Consoli A, Condette-Auliac S, Coskun O, Di Maria F, Rodesch G. Concomitant conus medullaris arteriovenous shunts and sacral dural arteriovenous fistulas: Pathophysiological links related to the venous drainage of the lesions in a series of five cases. J Neurointerv Surg 2018; 10(6):586-92. doi: 10.1136/neurintsurg- 2017-013505.

4. Sharma P, Ranjan A, Lath R. Arteriovenous fistula of the filum terminale misdiagnosed and previously operated as lower lumbar degenerative disease. Asian spine J 2014; 8(3):365-70. doi: 10.4184/asj.2014.8.3.365. 
Guiyun Zhang and Fei Shi

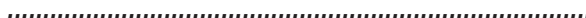

Department of Neurosurgery, Shanghai General Hospital, Shanghai Jiao Tong University School of Medicine, Shanghai, China

Correspondence to: Dr. Fei Shi, Department of Neuro- surgery, Shanghai General Hospital, Shanghai Jiao Tong University School of Medicine, Shanghai, China

E-mail: chobit39@sina.com

Received: February 14, 2020; Revised: April 21, 2020; Accepted: May 09, 2020

DOI: https://doi.org/10.29271/jcpsp.2021.01.117 